\title{
GÊNERO Baccharis (ASTERACEAE): ASPECTOS QUÍMICOS, ECONÔMICOS E BIOLÓGICOS
}

\author{
Luiz Gonzaga Verdi, Inês Maria Costa Brighente e Moacir Geraldo Pizzolatti*
}

Departamento de Química, Universidade Federal de Santa Catarina, CP 476, 88040-900 Florianópolis-SC

Recebido em 17/11/03; aceito em 17/5/04; publicado na web em 9/9/04

\begin{abstract}
THE Baccharis GENUS (ASTERACEAE): CHEMICAL, ECONOMIC AND BIOLOGICAL ASPECTS. The Baccharis genus is represented by more than 500 species distributed mainly in the tropical areas of South America. Many of them are extensively used in folk medicine in the treatment or prevention of anemias, inflammations, diabetes and stomach, liver and prostate diseases. Phytochemical and biological investigations in about 120 species resulted mainly in the isolation of clerodane and labdane diterpenes and flavonoid aglicones with the flavone unit being the most frequent.
\end{abstract}

Keywords: Baccharis; flavonoids; terpenes.

\section{INTRODUÇÃO}

A família Asteraceae é o grupo sistemático mais numeroso dentro das Angiospermas, compreendendo cerca de 1.100 gêneros e 25.000 espécies. São plantas de aspecto extremamente variado, incluindo principalmente pequenas ervas ou arbustos e raramente árvores $^{1}$. Cerca de $98 \%$ dos gêneros são constituídos por plantas de pequeno porte, e são encontradas em todos os tipos de habitats, mas principalmente nas regiões tropicais montanhosas na América do $\mathrm{Sul}^{2}$.

Plantas dessa família são extensivamente estudadas quanto a sua composição química e atividade biológica, sendo que algumas têm proporcionado o desenvolvimento de novos fármacos, inseticidas, entre outros ${ }^{3}$. Dentre inúmeras plantas da família Asteraceae utilizadas na medicina caseira está a Artemisia absinthium, uma erva de sabor amargo conhecida popularmente como losna, com benéficas funções digestivas, usada também na fabricação da bebida absinto ${ }^{2}$. Inúmeros trabalhos científicos realizados com espécies da família Asteraceae apresentaram o isolamento de uma variedade de metabólitos secundários com destaque aos flavonóides, alocados como importantes marcadores quimiotaxonômicos ${ }^{4}$, além de sua reconhecida importância para a medicina, no tratamento e prevenção de várias doenças ${ }^{5}$.

O gênero Baccharis (tribo Astereae) está representado por mais de 500 espécies distribuídas principalmente no Brasil, Argentina, Colômbia, Chile e México, ocupando as regiões mais elevadas ${ }^{6,7}$. A alta concentração de espécies no Brasil e nos Andes indica que uma dessas áreas é o provável centro de origem desse gênero ${ }^{8}$. No Brasil estão descritas 120 espécies de Baccharis, com a maior parte delas localizadas na região sudeste do País ${ }^{9}$. Estima-se em 100 as espécies na Argentina ${ }^{10}, 28$ no México ${ }^{11}$ e cerca de 40 na Colômbia, constituindo um dos mais importantes grupos de plantas neste país ${ }^{12,13}$, das quais $38 \%$ são endêmicas ${ }^{14,15}$.

As espécies do gênero Baccharis são no geral arbustos como a carqueja, a vassoura ou vassourinha e medem em média de 0,5 a 4,0 m de altura. Apresentam elevado valor sócio-econômico, com ampla dispersão nos estados de Santa Catarina, Paraná, São Paulo e Rio Grande do Sul, entre outras regiões do país, onde grande número delas são utilizadas na medicina popular para controle ou tratamento de várias doenças. São consumidas principalmente na forma

\footnotetext{
*e-mail: mgpizzo@qmc.ufsc.br
}

de chás com indicações para males do estômago, fígado, anemias, inflamações, diabetes, doenças na próstata, sendo também descritas como remédio para o processo de desintoxicação do organismo ${ }^{16-18}$. Por exemplo, no Brasil e Argentina , B. crispa e a B. notosergila são usadas para curar feridas e inflamações ${ }^{19}$. Outras espécies bastante reconhecidas na medicina alternativa são as $B$. trimera e $B$. articulata $^{20}$. B. genistelloides é uma erva medicinal muito usada no Brasil para uma variedade de doenças, tais como desordens digestivas e do fígado, malária, úlceras, diabetes, anemia, diarréia, inflamações urinárias, amigdalite, verminoses, mal de Hansen, entre outras ${ }^{21}$.

O estudo de espécies do gênero Baccharis tem mostrado grandes avanços devido ao seu reputado uso na medicina caseira na América Latina. Sua fitoquímica destaca a ocorrência de flavonóides, diterpenos e triterpenos, sendo nitidamente observado maior acúmulo de flavonas, flavonóis e de diterpenos labdanos e clerodanos ${ }^{22,23}$.

\section{ASPECTOS ECONÔMICOS}

Muitas são as implicações econômicas dessas espécies, podendo-se citar aspectos negativos e positivos. Por exemplo, três arbustos do sudeste dos Estados Unidos, B. neglecta, B. halimifolia e $B$. salicifolia causaram impactos negativos para a economia. $B$. neglecta é uma planta invasiva, afetando as pastagens em algumas regiões do Texas, enquanto a $B$. halimifolia é tóxica para o gado, causando sintomas como tontura, tremores, convulsão, diarréia e outros transtornos gastrointestinais. B. salicifolia é freatófita, a qual torna inútil a valiosa água no seco sul dos Estados Unidos ${ }^{24}$. Em pastagens brasileiras têm-se registros de envenenamento de animais devido à ingestão de $B$. megapotamica e $B$. coridifolia, espécies que acumulam tricotecenos, substâncias altamente tóxicas ${ }^{25,26}$. Estas substâncias são responsáveis por numerosos casos de intoxicações, podendo causar a morte em animais e seres humanos ${ }^{27}$. B. coridifolia é um problema freqüente na América do Sul, sendo uma das plantas mais tóxicas para o gado no Brasil, Argentina e Uruguai ${ }^{28}$. Estudos mostraram que 0,25 a $0,50 \mathrm{~g}$ da planta por $\mathrm{kg}$ do animal, quando estas estão com flores, ou $2 \mathrm{~g} / \mathrm{kg}$ de talos da planta em crescimento é suficiente para levar à morte dentro de $14-41$ h após a ingestão ${ }^{28}$. B. coridifolia e $B$. artemisioides, bastante comuns na Argentina, também causam problemas para os animais ${ }^{29}$. Algumas espécies trazem benefícios, como B. pluralis que é bastante utilizada na delimitação de terrenos na forma de cerca viva e na cobertura de solo para prevenir erosão e, também, para evitar poeira. $B$. micrantha é usada na Colômbia para 
proteger o solo contra erosão ${ }^{30}$. As espécies B. salicifolia e B. pluralis, durante a floração são grandes atrativos de abelhas pois o néctar leva à produção de mel com excelente qualidade. Por outro lado, as espécies $B$. angustifolia, B. glomeruliflora e B. neglecta são usadas como plantas ornamentais ${ }^{31}$. A partir das folhas da $B$. dracunculifolia e $B$. genistelloides do sudeste do Brasil são extraídos, por arraste a vapor, óleo de vassoura e óleo de carqueja, de alto valor para a indústria de fragrância ${ }^{32}$.

\section{PRINCIPAIS CONSTITUINTES DO GÊNERO Baccharis: OCORRÊNCIA E PROPRIEDADES BIOLÓGICAS}

Cerca de 120 espécies do gênero Baccharis foram estudadas quimicamente e entre estas, em torno de 30 apresentam estudos de atividade biológica que estão sumarizados na Tabela 1. De modo geral, os compostos que mais se destacam são os flavonóides, clerodanos e labdanos, embora também se tenha obsevado com certa frequiência a presença de kauranos, triterpenos, germacreno, ácidos cumáricos, tricotecenos, sesquiterpenos e fenilpropanóides. Na Tabela 2 é apresentada uma panorâmica da relação flavonóides e terpenóides, grupos de maior ocorrência no gênero Baccharis. Nos estudos de atividades biológicas destacam-se os efeitos alelopáticos, antimicrobianos, citotóxicos e antiinflamatórios. Entre as espécies mais pesquisadas quanto à composição química e/ou atividade biológica, encontram-se $B$. megapotamica, $B$. incarum, $B$. trimera, $B$. trinervis, B. salicifolia, B. crispa, B. coridifolia, B. dracunculifolia, B. grisebachii e B. tricuneata.

\section{Terpenóides}

Os tricotecenos eram conhecidos, até pouco tempo, como metabólitos produzidos exclusivamente por fungos, principalmente dos

Tabela 1. Atividades biológicas de extratos e compostos isolados de espécies do gênero Baccharis

\begin{tabular}{|c|c|c|c|}
\hline Espécie & Compostos/Extratos & Bioatividade & Ref. \\
\hline B. artemisioides & clerodanos & "antifeedant", & 77 \\
\hline B. articulata & extrato & antioxidante & 92 \\
\hline B. boliviensis & extrato e ácido ferrúlico & alelopática & 62 \\
\hline B. conferta & flavonóides, triterpeno & espasmolítica, antibacteriana & 61 \\
\hline \multirow[t]{4}{*}{ B. coridifolia } & tricotecenos & antiviral e aleloquímica & 86,71 \\
\hline & extrato & antioxidante & 91,66 \\
\hline & & citotóxica & 90,66 \\
\hline & & inseticida & 78 \\
\hline \multirow[t]{3}{*}{ B. crispa } & clerodanos & "antifeedant" & 51,77 \\
\hline & flavonóides & antimicrobiana & 58 \\
\hline & extrato & antioxidante & 91 \\
\hline B. gaudichaudiana & clerodanos, labdanos e flavonóides & citotóxica & 49 \\
\hline B. genistelloides & extrato & antiviral, gastroprotetiva & 72,75 \\
\hline B. grisebachii & extrato, diterpenos e der. ácido p-cumárico & $\begin{array}{l}\text { antimicrobiana, citotóxica, } \\
\text { e síntese proteica }\end{array}$ & $\begin{array}{l}60,70,73 \\
68,158\end{array}$ \\
\hline B. heterophylla & extrato & antimicrobiana e espasmolítica & 83 \\
\hline B. illinita & extrato & gastroprotetiva & 99 \\
\hline \multirow[t]{2}{*}{ B. incarum } & extrato & síntese proteica & 68 \\
\hline & & antibacteriana, antiinflamatória & 70,81 \\
\hline B. latifolia & extrato & síntese proteica & 68 \\
\hline B. lejia & clerodanos & citotóxica & 50 \\
\hline \multirow[t]{3}{*}{ B. linearis } & ácido oleanólico & repelente a insetos fitófagos & 76 \\
\hline & extrato, terpenos e & alelopática & 63 \\
\hline & flavonóides & citotóxica e "antifeedant" & 84 \\
\hline B. magellanica & extrato e acetofenonas & alelopática & 63 \\
\hline \multirow[t]{4}{*}{ B. megapotamica } & tricotecenos & aleloquímica, toxidade cerebral & $71,69,79,80$ \\
\hline & & antileucêmica & 23,34 \\
\hline & & antibiótica & 67 \\
\hline & saponinas, flavonóides & antiinflamatória e analgésica & 55 \\
\hline B. notosergila & flavonóides, óleo essencial & antimicrobiana & 58,59 \\
\hline B. ochracea & extrato & citotóxica & 90 \\
\hline B. pedunculata & lactonas, cumarinas e flavonóides & antifúngica & 53 \\
\hline B. pseudotenuifolia & extrato e flavonóide & citotóxica & 87 \\
\hline$B$. rethinoides & clerodanos & "antifeedant" & 77 \\
\hline B. rubricaulis & extrato & gastroprotetiva, antiviral & 72,75 \\
\hline B. sagittalis & clerodanos & "antifeedant" & 82 \\
\hline B. sarothroides & flavonóides & citotóxica & 54 \\
\hline B. serraefolia & extrato & antiespasmódica & 74 \\
\hline B. teindalensis & extrato & antiviral & 88 \\
\hline B. triangularis & clerodanos & "antifeedant" & 77 \\
\hline \multirow[t]{4}{*}{ B. trimera } & clerodano e flavonóide & vasorelaxante & 52 \\
\hline & flavonóides & moluscicida & 85 \\
\hline & & antimutagênica & 56 \\
\hline & & hepatoprotetora & 57 \\
\hline \multirow[t]{2}{*}{ B. trinervis } & extrato & antiviral & 64 \\
\hline & & antiinflamatória e antioxidante & 65 \\
\hline B. umbelliformis & extrato e acetofenonas & alelopática & 63 \\
\hline
\end{tabular}


Tabela 2. Ocorrência de flavonóides e terpenos por espécie no gênero Baccharis

\begin{tabular}{|c|c|c|c|c|c|c|c|}
\hline Espécies & Flavona & Flavanona & Kaurano & Labdano & Clerodano & Triterpeno & Ref. \\
\hline B. alaternoides & 1 & 3 & & & 2 & 1 & 118,133 \\
\hline B. angustifolia & 1 & & & & & & 131 \\
\hline B. artemisioides $^{*}$ & & & & & 2 & & 135,136 \\
\hline B. articulata $^{*}$ & & 5 & & & 4 & & $101,137,138$ \\
\hline B. bigelovii & 13 & 5 & & & & & $43,95,111$ \\
\hline B. boliviensis & & & & 4 & 16 & 1 & 48 \\
\hline B. calvescens ${ }^{*}$ & & & & & 1 & 3 & 46 \\
\hline B. chilco & & & & & & 2 & 44 \\
\hline B. cassinaefolia* & & & & & & 2 & 46 \\
\hline B. concata & 1 & & & 1 & 1 & 2 & 97 \\
\hline B. concinna & & 1 & 3 & & & 2 & 47 \\
\hline B. conferta & 3 & 1 & & & & 3 & 21,61 \\
\hline B. confertifolia & 1 & 5 & & & & & 97 \\
\hline B. crispa* & 3 & & & & 5 & & $19,51,58,139$ \\
\hline B. decussata & 2 & & & & & & 94 \\
\hline B. dracunculifolia* & 2 & 1 & & & & 1 & 48,159 \\
\hline B. eggersii & & & & 2 & & 1 & 44 \\
\hline B. eleagnoides & 3 & & & & & & 116 \\
\hline B. flabellata & 4 & & & & 7 & 1 & $45,103,104$ \\
\hline B. gaudichaudiana* & 9 & & & 9 & 2 & & $49,96,140,141$ \\
\hline B. genistelloides ${ }^{*}$ & 10 & 1 & & & 10 & & $105,108,115$ \\
\hline B. gilliesii & 1 & & & & & & 101 \\
\hline B. glutinosa* & & 1 & & & & & 134 \\
\hline B. grandicapitulata & 2 & & & & 1 & 3 & 44 \\
\hline B. grisebachii & 3 & & & 2 & & & 158 \\
\hline B. halimifolia* & 1 & & & & 4 & & 43 \\
\hline B. heterophylla & 2 & 1 & & & 2 & 2 & $43,95,142$ \\
\hline B. hutchisonii & & & & & 2 & 2 & 44 \\
\hline B. illinita & 9 & 3 & 3 & & & & 157 \\
\hline B. incarum & 2 & & & & 9 & 2 & $128,143,144$ \\
\hline B. intermixta* & & 1 & 2 & & & & 46 \\
\hline B. kingii & 1 & & & & 1 & & 121 \\
\hline B. latifolia & & & & 3 & & 1 & 47,48 \\
\hline B. lejía & 4 & & & & 16 & 1 & 50 \\
\hline B. leptocephala* & & 2 & & & & 1 & 47 \\
\hline B. ligustrina* & 1 & 1 & & & & 2 & 109 \\
\hline B. linearis & 4 & & & & 7 & 3 & $76,84,106,145$ \\
\hline B. macraei & & & & & 8 & & 146,147 \\
\hline B. magellanica* & 1 & & & & 2 & 2 & $42,100,107$ \\
\hline B. marginalis & & 1 & & & 1 & & 113 \\
\hline B. maritima* & 2 & & & & & & 112 \\
\hline B. medullosa & 1 & & & 2 & & & 104,148 \\
\hline B. microcephala* & 2 & & & & 1 & & 44 \\
\hline B. minutiflora & & & 8 & & & 2 & 118 \\
\hline B. myrsinites & & & & 2 & & 2 & 149 \\
\hline B. neaei & 2 & & & 5 & & & 119 \\
\hline B. neglecta* & & & & & 3 & 2 & 43 \\
\hline B. nitida & 2 & & & & & 1 & 44 \\
\hline B. notosergila* & 2 & & & & & & 58 \\
\hline B. obtusifolia & 1 & & & & 3 & 1 & 48 \\
\hline B. ochracea* & 1 & & & & & & 130 \\
\hline B. oxydonta* & & 2 & & 5 & & 3 & 47 \\
\hline B. paniculata & 2 & 2 & & 2 & & & 97 \\
\hline B. patagonica* & 2 & & & & 3 & 5 & 42,100 \\
\hline B. patens ${ }^{*}$ & 2 & & & & & & 129 \\
\hline B. pedicellata & 3 & & & & 1 & 2 & 113 \\
\hline B. pedunculata & 4 & & & & 4 & & 53,124 \\
\hline B. peruviana & 2 & & & & 2 & & 48 \\
\hline B. petiolata & 4 & 2 & & 6 & & & 97,120 \\
\hline B. pingraea & & & & 21 & & & $42,150,151$ \\
\hline
\end{tabular}


Tabela 2. continuação

\begin{tabular}{|c|c|c|c|c|c|c|c|}
\hline Espécies & Flavona & Flavanona & Kaurano & Labdano & Clerodano & Triterpeno & Ref. \\
\hline B.platypoda* & 2 & 6 & 1 & & & 2 & 152 \\
\hline B. polifolia & 2 & 1 & & 2 & & & 42 \\
\hline B. polyphylla & & & 5 & & & & 46 \\
\hline B. potosina & & 2 & 2 & 1 & & & 43 \\
\hline B. pseudotenuifolia ${ }^{*}$ & 8 & 3 & & & & 1 & 87 \\
\hline B. pteronioides & 7 & 1 & & 4 & 7 & & 43,95 \\
\hline B. pylicoides & & 2 & 1 & & & 1 & 44 \\
\hline B. quitensis & 4 & & 6 & & & 5 & 47,95 \\
\hline B. ramoisissima* & 1 & 1 & 2 & & & 6 & 46,47 \\
\hline B. reticularia ${ }^{*}$ & & 1 & & & & & 46 \\
\hline B. retusa* & & 1 & & & & & 122 \\
\hline B. rhetinoides & & & & & 2 & 1 & 42 \\
\hline B. rhomboidalis & 1 & & & & 9 & 1 & 126,153 \\
\hline B. sagittalis ${ }^{*}$ & & & & 1 & 8 & & 82 \\
\hline B. salicifolia & 15 & 7 & & 17 & 3 & 2 & $42,43,95$ \\
\hline B. salicina & 2 & & & & 4 & 2 & $43,98,99$ \\
\hline B. salzmannii ${ }^{*}$ & & 1 & 2 & & & 3 & 46 \\
\hline B. santelicis & & & & & 5 & & 120 \\
\hline B. sarothroides & 10 & & & & 7 & 1 & $43,54,95,142$ \\
\hline B. scoparia & & & & 2 & 3 & 3 & 44 \\
\hline B. serraluta* & & 1 & & & & & 46 \\
\hline B. sternbergiana & 2 & & & 6 & & 2 & 132 \\
\hline B. subdentata & & & & & 1 & 4 & 47 \\
\hline B. thesioides & 5 & 1 & & & & & 43,110 \\
\hline B. thymifolia & 2 & & & & & & 127 \\
\hline B. tola & 2 & & 10 & & & 1 & $154,114,125$ \\
\hline B. tricuneata & & 1 & & & & & 132 \\
\hline B. trimera* & 10 & & & & 3 & & $48,52,55,56,57,85,122$ \\
\hline B. trinervis ${ }^{*}$ & 10 & 1 & & & 24 & 2 & $47,93,102,123,133,155$ \\
\hline B. truncata & & 1 & 5 & & 1 & 3 & 47 \\
\hline B. tucumanensis & 2 & & & 4 & 1 & & 117,156 \\
\hline B. ulicina* & & & & & & 1 & 42 \\
\hline B. vaccinoides & 22 & 2 & & & 3 & 3 & 95,142 \\
\hline B. varians $^{*}$ & & 4 & & & & & 46 \\
\hline
\end{tabular}

* Espécies brasileiras

gêneros Fusarium e Myrothecium. Em 1976 este tipo de metabólito foi primeiramente detectado em plantas superiores, ao ser isolado de B. megapotamica. Inicialmente suspeitou-se de que estas plantas estavam contaminadas com fungos, ou que havia uma associação da planta com o fungo presente no solo. Jarvis e colaboradores, em seus estudos sobre a origem dos tricotecenos em Baccharis, demonstraram que tais metabólitos são biossintetizados em algumas espécies após a polinização das plantas fêmeas, como por exemplo em B. coridifolia $^{33}$. Esses diterpenos constituem uma nova classe de compostos potencialmente importantes no desenvolvimento de fármacos anticâncer e estão presentes principalmente em espécies coletadas na Argentina e nas regiões sul e sudeste do Brasil, Rio Grande do Sul, Paraná e São Paulo, com maior concentração nas plantas brasileiras. Por exemplo, o tricoteceno baccharin [1], isolado de B. megapotamica foi ativo contra leucemia implantada em ratos ${ }^{34,35}$. Os tricotecenos macrocíclicos roridin A [2], roridin E [3], verrucarin A [4] e verrucarin J [5] de B. coridifolia apresentaram atividade antiviral ${ }^{36}$. Ensaios de toxicidade in vivo realizados em coelhos e ratos mostram que com a ingestão de $B$. coridifolia e/ou tricotecenos purificados desenvolveram sintomas tóxicos que, muitas vezes, resultaram na morte dos animais, sendo a diarréia o sintoma mais comum ${ }^{37,38}$. Esses dois grupos de tricotecenos roridinas e verrucarinas, encontrados em $B$. coridifolia, têm mostrado atividade contra células $(\mathrm{KB})$ derivadas de carcinoma humano da nasofaringe $\mathrm{e}^{34,35}$. Entre as espécies de Baccharis estudadas apenas B. megapotamica, $B$. coridifolia e B. artemisioides contêm tricotecenos ${ }^{26,29,39-41}$.

Os diterpenos são os compostos encontrados em maior quantidade no gênero Baccharis e são representados principalmente por neo e ent-clerodanos e, menos comum, ent-labdanos e kauranos ${ }^{42-48}$. Contudo, apesar do grande número de compostos isolados e conhecidos, pouco se conhece sobre a atividade biológica dessas substâncias. Alguns compostos isolados de B. gaudichaudiana foram testados em células com leucemia linfocítica P-388, bem como em uma sequiência de linhagem de células humanas. Entre os diterpenóides testados, os labdanos gaudichaudols A-C $[\mathbf{6}, \mathbf{7}, \mathbf{8}]$, os clerodanos gaudichaudona [9] e acetato de articulina [10] mostraram significante atividade citotóxica contra algumas células testadas ${ }^{49}$. A partir das folhas de B. lejia foram isolados uma série de neo-clerodanos com importante toxicidade para Artemia salina. Esta toxicidade foi constatada apenas nos neo-clerodanos contendo um grupo hidroxila livre em $\mathrm{C}-18^{50}$. Outros diterpenóides clerodanos das espécies $B$. rethinoides, $B$. triangularis, $B$. artemisioides e $B$. crispa apresentaram atividade inseto repelente e "antifeedant" (inibição da vontade de se alimentar $)^{37,51}$. O diterpeno clerodano dilactônico [11] de $B$. trimera apresentou efeito vasorelaxante para a musculatura lisa 
vascular em $\operatorname{ratos}^{52}$. Esse efeito pode estar relacionado à vasodilatação e melhora da circulação sanguínea constatada pela medicina popular para esta planta ${ }^{18}$

Os triterpenos apresentam 103 ocorrências em 48 espécies de Baccharis, Tabela 2, com 23 compostos diferentes sendo representados princialmente pelo ácido oleanólico, que foi encontrado em 24 espécies e óxido de baccharis [12], encontrado em 17 espécies. Dentre as raras atividades biológicas de Baccharis atribuídas aos triterpenos, enfatizamos a atividade repelente a insetos fitófagos do ácido oleanólico isolado de $B$. linearis ${ }^{76}$.
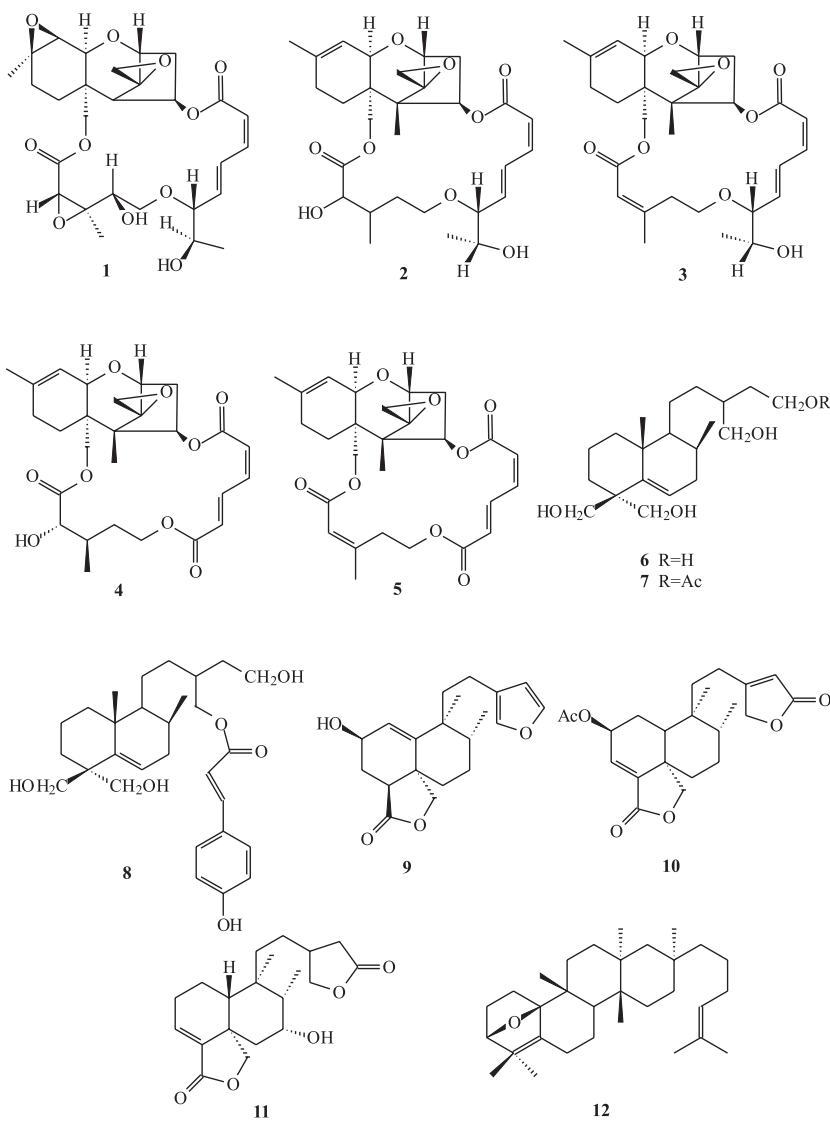

\section{Flavonóides}

Os flavonóides, juntamente com os diterpenos, são os compostos de maior ocorrência no gênero Baccharis (Tabela 2) e são descritos como bons marcadores quimiotaxonômicos para os mais baixos níveis hierárquicos da família Asteraceae ${ }^{4}$. Apresentam-se normalmente como agliconas livres e muito raramente na forma glicosilada, o que é uma característica da família Asteraceae. São 298 ocorrências de flavonóides no gênero Baccharis com 109 compostos diferentes, sendo 24 com unidade flavanona e 85 com unidade flavona, das quais $48 \%$ apresentam-se oxigenadas em C-3 (Tabela 3). O padrão de oxigenação mais frequente ocorre em C-5 e C-7 do anel A e C-4' do anel B, o grupo metoxila aparece com maior freqüência em C-7 e C-6 do anel A e C-4'do anel B.

Os testes de atividade biológica realizados em extratos brutos e frações de plantas do gênero Baccharis mostraram que a maioria das atividades não estão relacionadas aos flavonóides e sim aos terpenos e tricotecenos. Todavia, os flavonóides estão despertando muito interesse em diversas aplicações para a medicina, entre elas como antioxidantes, possuindo diferentes graus de atividade em diferentes modelos experimentais.
Compostos de $B$. pedunculata, incluindo dois flavonóides, 5,7diidroxi-6,4'-dimetoxiflavona (pectolinaringenina) [13] e 5,4'diidroxi-3,6,7-trimetoxiflavona (penduletina) [14] foram testados para atividade antifúngica, sendo o composto [13] responsável pela atividade $^{53}$. Dois flavonóides citotóxicos 3',5,7-triidroxi-3,4'dimetoxiflavona (tamarixetina-3,4'-dimetil eter) [15] e 5,7,3'triidroxi-3,6,4'-trimetoxiflavona (centaureidina) [16] foram isolados de $B$. sarothroides ${ }^{54}$. B. trimera destaca-se por sua importante atividade antiinflamatória e analgésica, atribuída principalmente às saponinas, sendo que o flavonóide rutina mostrou-se inativo ${ }^{55}$. A partir de $B$. trimera (carqueja), usada na medicina popular para o tratamento de doenças do fígado e reumatismo, foram isolados do extrato metanólico das folhas, quatro flavonóides, 5,4'-diidroxi-7metoxiflavona (genkwanina) [17], 5,4'-diidroxi-6,7-dimetoxiflavona (cirsimaritina) [18], 5,7,4'-triidroxi-6-metoxiflavona (hispidulina) [19] e 5,7,4'-triidroxiflavona (apigenina) [20]. Estes compostos apresentaram atividade antimutagênica, mostrando que tais flavonas são as substâncias responsáveis pela atividade na carqueja. Os testes mostraram certa tendência na diminuição da atividade quando os grupos hidroxilas foram substituídos pelos grupos metoxilas nessas flavonas ${ }^{56}$. A partir do extrato em acetato de etila dessa mesma planta, outros três flavonóides foram isolados, 3,5,7,3',4'-pentaidroxiflavona (quercetina) [21], 5,7,3',4'-tetraidroxiflavona (luteolina) [22] e 5,7,3',4'-tetraidroxi-6-metoxiflavona (nepetina) [23] e determinadas suas propriedades anti-hepatotóxica, utilizando ratos como modelo. Dos compostos, o mais ativo foi a hispidulina, mas o extrato bruto e uma fração enriquecida com os cinco flavonóides teve resultados ainda melhores ${ }^{57}$. Os flavonóides genkwanina e apigenina, isolados de B. crispa e B. notosergila, juntamente com seus extratos etanólico e óleo essencial, apresentaram atividade antibacteriana, que também foi observada nas espécies $B$. rufescens e $B$. medulosa, justificando o uso dessas plantas na medicina popular para a cura de feridas e infecções microbianas ${ }^{58-60}$. Extratos de $B$. conferta e alguns compostos isolados, entre eles os flavonóides apigenina-4',7-dimetil eter [24], naringenina-4',7-dimetil eter [25], pectolinaringenina [13] e cirsimaritina [18] apresentaram ação espasmolítica ${ }^{61}$. Estes dados estão de acordo com a utilização desta planta no tratamento de cólicas gastrointestinais.<smiles>[R9]c1ccc(-c2oc3cc([R6])c([R9])c(O)c3c(=O)c2[R])cc1[R9]</smiles>

Flavona<smiles>COc1ccc(C2CC(=O)c3c(O)cc(OC)cc3O2)cc1</smiles>

25

Flavanona

$\begin{array}{lllllll} & \mathrm{R}_{1} & \mathrm{R}_{2} & \mathrm{R}_{3} & \mathrm{R}_{4} & \mathrm{R}_{5} & \mathrm{R}_{6} \\ 13 & \mathrm{H} & \mathrm{H} & \mathrm{MeO} & \mathrm{H} & \mathrm{H} & \mathrm{Me} \\ 14 & \mathrm{MeO} & \mathrm{H} & \mathrm{MeO} & \mathrm{Me} & \mathrm{H} & \mathrm{H} \\ 15 & \mathrm{MeO} & \mathrm{H} & \mathrm{H} & \mathrm{H} & \mathrm{OH} & \mathrm{Me} \\ 16 & \mathrm{MeO} & \mathrm{H} & \mathrm{MeO} & \mathrm{H} & \mathrm{OH} & \mathrm{Me} \\ 17 & \mathrm{H} & \mathrm{H} & \mathrm{H} & \mathrm{Me} & \mathrm{H} & \mathrm{H} \\ 18 & \mathrm{H} & \mathrm{H} & \mathrm{MeO} & \mathrm{Me} & \mathrm{H} & \mathrm{H} \\ 19 & \mathrm{H} & \mathrm{H} & \mathrm{MeO} & \mathrm{H} & \mathrm{H} & \mathrm{H} \\ 20 & \mathrm{H} & \mathrm{H} & \mathrm{H} & \mathrm{H} & \mathrm{H} & \mathrm{H} \\ 21 & \mathrm{OH} & \mathrm{H} & \mathrm{H} & \mathrm{H} & \mathrm{OH} & \mathrm{H} \\ 22 & \mathrm{H} & \mathrm{H} & \mathrm{H} & \mathrm{H} & \mathrm{OH} & \mathrm{H} \\ 23 & \mathrm{H} & \mathrm{H} & \mathrm{MeO} & \mathrm{H} & \mathrm{OH} & \mathrm{H} \\ 24 & \mathrm{H} & \mathrm{H} & \mathrm{H} & \mathrm{Me} & \mathrm{H} & \mathrm{Me}\end{array}$

Outras atividades relevantes são o efeito alelopático observado em B. boliviensis, B. linearis, B. magelanica e B. umbelliformis ${ }^{62,63} \mathrm{e}$ a atividade anti-HIV de $B$. trinervi ${ }^{64}$. Finalmente, observou-se a ati- 
Tabela 3. Flavonóides isolados de espécies do gênero Baccharis

\begin{tabular}{l} 
Compostos \\
\hline $\begin{array}{l}\text { 5,7-OH-6,4'-OMe flavona } \\
\text { (pectolinaringenina) }\end{array}$ \\
\\
5,7-OH-6,3',4'-OMe flavona \\
(eupatilina) \\
5,7,3'-OH-3,6,4'-OMe flav \\
(centaureidina) \\
5,7,3'-OH-6,4'-OMe flavona \\
(desmetoxicentaureidina) \\
5,7-OH-2'-OMe flavona \\
(2'-metoxicrisina) \\
5,7-OH-4'-OMe flavona \\
(acacetina)
\end{tabular}

5,7,4'-OH-6,3'-OMe flavona (jaceosidina)

5,3',4'-OH-6,7-OMe flavona (cirsiliol)

5,7,3',4'-OH-6-OMe flavona (nepetina/eupafolina)

5,7,4'-OH-6-OMe flavona (hispidulina)

$3,5,7,3^{\prime}, 4^{\prime}-\mathrm{OH}$ flavona (quercetina)

5,4'-OH-3,6,7-OMe flavona (penduletina)

$5,7,3^{\prime}, 4^{\prime}-\mathrm{OH}$ flavona (luteolina)

5,7,4'-OH flavona (apigenina)

B. illinita 157

B. grandicapitulata 44

B. vaccinoides 95

B. salicifolia 95

B. articulata 101

B. trinervis 102

B. gaudichaudiana 96

B. genistelloides 105

B. flabellata 103

B. gaudichaudiana 96

B. trimera $\quad 57$

B. linearis $\quad 106$

B. flabellata 103

B. halimifolia 43

B. vaccinoides 95

B. ligustrina 109

B. thesioides $\quad 110$

B. trimera 57

B. illinita 157

B. salicifolia 95

B. pseudotenuifolia 87

B. pedunculata 53

B. salicifolia 95

B. vaccinoides 95

B. sarothroides 95

B. trinervis 93

B. microcephala 44

B. nitida

B. bigelovii

B. illinita

B. pteronioides

B. trimera

B. gaudichaudiana

\begin{tabular}{lc} 
Espécies & Ref. \\
\hline B. pedunculata & 53 \\
B. grisebachii & 158 \\
B. trinervis & 93 \\
B. decussata & 94 \\
B. vaccinoides & 95 \\
B. conferta & 61 \\
B. gaudichaudiana & 96 \\
& \\
B. sarothroides & 54
\end{tabular}

B. gaudichaudiana $96 \quad$ 5,4'-OH-7-OMe flavona

B. petiolata $97 \quad$ (genkwanina)

B. salicina $\quad 98,99$

B. patagonica $\quad 100,42$

B. flabellata 103, 104

B. flabellata 103, 104

B. magellanica 100,107

B. genistelloides 108

B. gaudichaudiana 49,96

B. trimera 56, 57

B. pseudotenuifolia 87

B. pteronioides $\quad 43,95$

B. bigelovii $\quad 95,111$

B. genistelloides 108

B. maritima 112

B. pedicellata

B. illinita

B. crispa

B. notosergila

B. trimera

B. genistelloides

B. bigelovii

Compostos
5,7,4'-OH flavona (apigenina)

5,3'-OH-6,7,4'-OMe flavona (eupatorina)

3,5,7-OH-4'-OMe flavona

(canferide)

5,4'-OH-6,7-OMe flavona (cirsimaritina)

5,4'-OH-6,7,8-OMe flavona (xantomicrol)

4'-OH-3,6,7,8,3'-OMe flavona 5,7,4'-OH-3,6,8,3'-OMe flavona $5,7,4$ '-OH-3,8,3'-OMe flavona 5-OH-7, 3', 4', 5' -OMe flavona (corimbosina)

5,7,3'-OH-4'-OMe flavona (diosmetina)

5,7-OH flavona (crisina)

3,5,7-OH flavona (galangina)

5,4'-OH-6,7,3'-OMe flavona (eupatrina)

95, 111

157

95

57

49

113

157

58

58

56,57

105

95,111

\begin{tabular}{|c|c|}
\hline Espécies & Ref. \\
\hline B. heterophylla & 95 \\
\hline B. pteronioides & 95 \\
\hline B. vaccinoides & 95 \\
\hline B. ramosissimum & 47 \\
\hline B. tola & 114 \\
\hline B. trinervis & 102 \\
\hline B. salicifolia & 95 \\
\hline B. pseudotenuifolia & 87 \\
\hline B. trimera & 52,85 \\
\hline B. genistelloides & 115 \\
\hline B. trinervis & 102 \\
\hline B. articulata & 101 \\
\hline B. medulosa & 104 \\
\hline B. vaccinoides & 95 \\
\hline B. heterophylla & 95 \\
\hline B. genistelloides & 105 \\
\hline B. trimera & 56 \\
\hline B. eleagnoides & 116 \\
\hline B. patagonica & 42 \\
\hline B. pedicellata & 113 \\
\hline B. notosergila & 58 \\
\hline B. pteronioides & 95 \\
\hline B. grandicapitulata & 44 \\
\hline B. salicifolia & 95 \\
\hline B. vaccinoides & 95 \\
\hline B. genistelloides & 105,115 \\
\hline B. trimera & 56 \\
\hline B. concata & 97 \\
\hline B. conferta & 61 \\
\hline B. vaccinoides & 95 \\
\hline B. articulata & 101 \\
\hline B. eleagnoides & 116 \\
\hline B. pteronioides & 95 \\
\hline B. vaccinoides & 95 \\
\hline B. bigelovii & 95,111 \\
\hline B. illinita & 157 \\
\hline B. salicifolia & 95 \\
\hline B. sarothroides & 95 \\
\hline B. maritima & 112 \\
\hline B. genistelloides & 108 \\
\hline B. pseudotenuifolia & 87 \\
\hline B. tucumanensis & 117 \\
\hline B. quitensis & 47,95 \\
\hline B. lejia & 50 \\
\hline B. lejia & 50 \\
\hline B. lejia & 50 \\
\hline B. salicifolia & 42 \\
\hline B. bigelovii & 95,111 \\
\hline B. salicifolia & 95 \\
\hline B. bigelovii & 43,95 \\
\hline B. bigelovii & 95 \\
\hline B. genistelloides & 105 \\
\hline B. decussata & 94 \\
\hline B. grisebachii & 158 \\
\hline B. polifolia & 42 \\
\hline B. bigelovii & 111 \\
\hline B. vaccinoides & 95 \\
\hline B. salicifolia & 95 \\
\hline B. alaternoides & 118 \\
\hline B. bigelovii & 95,111 \\
\hline B. illinita & 157 \\
\hline
\end{tabular}


Tabela 3. continuação

\begin{tabular}{|c|c|c|}
\hline Compostos & Espécies & Ref. \\
\hline \multirow{5}{*}{$\begin{array}{l}\text { 5,7,4'-OH-3-OMe flavona } \\
\text { (crisoeriol) }\end{array}$} & B. pteronioides & 95 \\
\hline & B. pseudotenuifolia & 87 \\
\hline & B. sarothroides & 95 \\
\hline & B. vaccinoides & 95 \\
\hline & B. linearis & 84 \\
\hline \multirow{5}{*}{$\begin{array}{l}5,4 \text { '-OH-3,7-OMe flavona } \\
\text { (kumatakenina) }\end{array}$} & B. sarothroides & 95 \\
\hline & B. neaei & 119 \\
\hline & B. pedicellata & 113 \\
\hline & B. petiolata & 120 \\
\hline & B. vaccinoides & 95 \\
\hline \multirow[t]{2}{*}{ 5,7,4'-OH-3,3'-OMe flavona } & B. kingii & 121 \\
\hline & B. linearis & 84 \\
\hline 5,7,3'-OH-3,4'-OMe flavona & B. sarothroides & 54 \\
\hline $\begin{array}{l}\text { 3,5,4'-OH-7-OMe flavona } \\
\text { (ramnocitrina) }\end{array}$ & B. vaccinoides & 95 \\
\hline \multirow[t]{2}{*}{ 8-OH flavona } & B. obtusifolia & 48 \\
\hline & B. dracunculifolia & 48 \\
\hline $\begin{array}{l}5,3^{\prime}-\mathrm{OH}-6,7,4 \text { '-OMe flavona } \\
\text { (eupatorina) }\end{array}$ & B. trimera & 48,122 \\
\hline 5-OH-3,7,4'-OMe flavona & B. petiolata & 120 \\
\hline \multirow[t]{2}{*}{ 5-OH-7,3',4'-OMe flavona } & B. salicifolia & 42 \\
\hline & B. trinervis & 123 \\
\hline 5-OH-6,7,3',4',5'-OMe flavona & B. salicifolia & 42 \\
\hline 5-OH-6,7,3',4'-OMe flavona & B. genistelloides & 115 \\
\hline 5-OH-6,7,4'-OMe flavona & B. trinervis & 93 \\
\hline \multirow[t]{2}{*}{ (salvigenina) } & B. articulata & 101 \\
\hline & B. pedunculata & 124 \\
\hline \multirow[t]{7}{*}{ 5-OH-7,4'-OMe flavona } & B. articulata & 101 \\
\hline & B. tola & 114,125 \\
\hline & B. rhomboidalis & 126 \\
\hline & B. trinervis & 102,123 \\
\hline & B. conferta & 31 \\
\hline & B. vaccinoides & 95 \\
\hline & B. crispa & 19 \\
\hline \multirow{2}{*}{ 5,7-OH-3',4'-OMe flavona } & B. trinervis & 102 \\
\hline & B. salicifolia & 95 \\
\hline \multirow[t]{2}{*}{ 5,3',4'-OH-7-OMe flavona } & B. microcephala & 44 \\
\hline & B. nitida & 44 \\
\hline \multirow{6}{*}{$\begin{array}{l}\text { 5,6-OH-7,4'-OMe flavona } \\
\text { (ladaneina) } \\
\text { 5,4'-OH-6,7,8,3'-OMe flavona } \\
\text { (7-Me-sudachitina) }\end{array}$} & B. tucumanensis & 117 \\
\hline & B. pedunculata & 124 \\
\hline & B. quitensis & 47 \\
\hline & B. lejia & 50 \\
\hline & B. thymifolia & 127 \\
\hline & B. incarum & 128 \\
\hline \multirow{4}{*}{$\begin{array}{l}5,7,3^{\prime}, 4^{\prime}-\mathrm{OH}-3-\mathrm{OMe} \text { flavona } \\
\text { (escoparol) } \\
5,3^{\prime}, 4^{\prime}-\mathrm{OH}-3,7-\mathrm{OMe} \text { flavona }\end{array}$} & B. linearis & 84,106 \\
\hline & B. illinita & 157 \\
\hline & B. neaei & 119 \\
\hline & B. paniculata & 97 \\
\hline \multirow[t]{2}{*}{ 5-OH-6,7,8,4'-OMe flavona } & B. polifolia & 42 \\
\hline & B. grisebachii & 158 \\
\hline 5-OH-6,7,8,3',4'-OMe flavona & B. thymifolia & 127 \\
\hline 7-OH-3,5,8-OMe flavona & B. eleagnoides & 116 \\
\hline 5,7-OH-3,6,3',4'-OMe flavona & B. salicina & 98 \\
\hline 5,4'-OH-3,6,7,8,3'-OMe flavona & B. incarum & 128 \\
\hline 5,3',4'-OH-6,7,8-OMe flavona & B. patens & 129 \\
\hline (sideritiflavona) & B. quitensis & 95 \\
\hline 5,3'-OH-7,4'-OMe flavona & B. crispa & 19 \\
\hline \multirow[t]{2}{*}{ (pilloina) } & B. confertifolia & 97 \\
\hline & B. trinervis & 123 \\
\hline \multirow{4}{*}{$\begin{array}{l}\text { 5,3'-OH-6,7,8,4'-OMe flavona } \\
\text { (gardenina D) } \\
\text { 3,5-OH-7,4'-OMe flavona }\end{array}$} & B. patens & 129 \\
\hline & B. quitensis & 95 \\
\hline & B. bigelovii & 95,111 \\
\hline & B. vaccinoides & 95 \\
\hline \multirow{3}{*}{$\begin{array}{l}\text { 3,5,7,4'-OH-3'-OMe flavona } \\
\text { (isoramnetina) }\end{array}$} & B. bigelovii & 95,111 \\
\hline & B. paniculata & 97 \\
\hline & B. petiolata & 97 \\
\hline
\end{tabular}

\begin{tabular}{|c|c|c|}
\hline Compostos & Espécies & Ref. \\
\hline \multirow{3}{*}{$\begin{array}{l}\text { 3,5,7,4'-OH-3'-OMe flavona } \\
\text { (isoramnetina) }\end{array}$} & B. pteronioides & 95 \\
\hline & B. salicifolia & 95 \\
\hline & B. pseudotenuifolia & 87 \\
\hline \multirow{3}{*}{$\begin{array}{l}5,7,3^{\prime}, 4^{\prime}-\mathrm{OH}-3-\mathrm{O}-\text { rutinosil } \\
\text { flavona (rutina) }\end{array}$} & B. thesioides & 110 \\
\hline & B. gaudichaudiana & 96 \\
\hline & B. trimera & 55 \\
\hline $\begin{array}{l}\text { 3,5,7-OH-3',4'-OMe flavona } \\
\text { (dillenetina) }\end{array}$ & B. salicifolia & 95 \\
\hline $5,7-\mathrm{OH}-3,3^{\prime}, 4^{\prime}-\mathrm{OMe}$ flavona & B. salicifolia & 95 \\
\hline \multirow{2}{*}{ 5,7,4'-OH-3,6-OMe flavona } & B. sarothroides & 95 \\
\hline & B. vaccinoides & 95 \\
\hline 5,7,4'-OH-3,8-OMe flavona & B. sarothroides & 95 \\
\hline $5,7,4^{\prime}-\mathrm{OH}-3,6,8-\mathrm{OMe}$ flavona & B. sarothroides & 95 \\
\hline 5,4'-OH-3,6,7,8-OMe flavona & B. sarothroides & 95 \\
\hline 5-OH-3,7,4'-OMe flavona & B. vaccinoides & 95 \\
\hline 3,5,7,4'-OH-6-OMe flavona & B. vaccinoides & 95 \\
\hline $\begin{array}{l}3,5,4^{\prime}-\mathrm{OH}-6,7-\mathrm{OMe} \text { flavona } \\
\text { (eupalitina) }\end{array}$ & B. vaccinoides & 95 \\
\hline 3,5,7-OH-6,4'-OMe flavona & B. vaccinoides & 95 \\
\hline (betuletol) & B. dracunculifolia & 159 \\
\hline 5,6-OH-3,7,4'-OMe flavona & B. vaccinoides & 95 \\
\hline 5-OH-3,6,7,4'-OMe flavona & B. vaccinoides & 95 \\
\hline 5,7,3',4'-OH-mono e diMe flavona & B. peruviana & 48 \\
\hline $\begin{array}{l}5,6,7,8,3^{\prime}, 4^{\prime} \text {-OMe flavona } \\
\text { (nobiletina) }\end{array}$ & B. illinita & 157 \\
\hline $\begin{array}{l}5,6,7,8,4^{\prime} \text {-OMe flavona } \\
\text { (tangeretina) }\end{array}$ & B. illinita & 157 \\
\hline \multirow{2}{*}{$\begin{array}{l}5,7,3^{\prime}, 4^{\prime} \text {-OH-3-O-glucosil flavona } \\
\text { (isoquercitrina) }\end{array}$} & B. thesioides & 110 \\
\hline & B. ochracea & 130 \\
\hline 5,7,3',4'-OH-3-O-glucosil flavona & B. pseudotenuifolia & 87 \\
\hline 5,7,3',4'-OH-3-O-ramnosil flavona & B. pseudotenuifolia & 87 \\
\hline (Quercetin-3-O-L- $\alpha$-ramnosideo) & B. gaudichaudiana & 96 \\
\hline $\begin{array}{l}5,7,3^{\prime}, 4^{\prime} \text {-OH-3-O-apiofuranosil- } \\
(1 \rightarrow 2 \text { )-galactosil flavona } \\
\text { (hiperosidina) }\end{array}$ & B. thesioides & 110 \\
\hline $\begin{array}{l}5,7,3^{\prime}, 4^{\prime}-\mathrm{OH}-3-\mathrm{O} \text {-apiofuranosil- } \\
(1 \rightarrow 2) \text {-ramno-piranosil } \\
(1 \rightarrow 6) \text {-glucopiranosil flavona }\end{array}$ & B. thesioides & 110 \\
\hline $\begin{array}{l}5,7,3^{\prime}, 4^{\prime}-\mathrm{OH}-3 \text {-O-ramnosil- } \\
(1 \rightarrow 6) \text {-glucosil flavona }\end{array}$ & B. trimera & 55 \\
\hline \multicolumn{3}{|l|}{$\begin{array}{l}\text { 8-glucopiranosil flavona } \\
\text { (isoscaftosida) }\end{array}$} \\
\hline 5,7,4'-OH-3-O-glucosil flavona & B. angustifolia & 131 \\
\hline \multicolumn{3}{|l|}{ (7-benzoil-crisina) } \\
\hline \multirow[t]{5}{*}{ 5-OH-7,4'-OMe flavanona } & B. salicifolia & 42 \\
\hline & B. confertifolia & 97 \\
\hline & B. pylicoides & 44 \\
\hline & B. conferta & 61 \\
\hline & B. sternbergiana & 132 \\
\hline 5-OH-7,3',4'-OMe flavanona & B. confertifolia & 97 \\
\hline 5,4'-OH-7-OMe flavanona & B. pylicoides & 44 \\
\hline \multirow[t]{13}{*}{ (sakuranetina) } & B. petiolata & 120 \\
\hline & B. paniculata & 97 \\
\hline & B. confertifolia & 97 \\
\hline & B. salicifolia & 42 \\
\hline & B. thesioides & 43 \\
\hline & B. trinervis & 123,133 \\
\hline & B. intermixta & 46 \\
\hline & B. tricuneata & 132 \\
\hline & B. sternbergiana & 132 \\
\hline & B. serraluta & 46 \\
\hline & B. retusa & 122 \\
\hline & B. leptocephala & 47 \\
\hline & B. pteronioides & 95 \\
\hline
\end{tabular}


Tabela 3. continuação

\begin{tabular}{|c|c|c|}
\hline Compostos & Espécies & Ref. \\
\hline \multirow[t]{2}{*}{ 5,4'-OH-7,3'-OMe flavanona } & B. salicifolia & 42 \\
\hline & B. confertifolia & 97 \\
\hline \multirow{5}{*}{$\begin{array}{l}5,7,3^{\prime}, 4^{\prime}-\mathrm{OH} \text { flavanona } \\
\text { (eriodictiol) }\end{array}$} & B. salicifolia & 42 \\
\hline & B. varians & 46 \\
\hline & B. reticularia & 46 \\
\hline & B. genistelloides & 105 \\
\hline & B. pseudotenuifolia & 87 \\
\hline \multirow[t]{6}{*}{ 5,7-OH flavanona (pinocembrina) } & B. oxydonta & 47 \\
\hline & B. concinna & 47 \\
\hline & B. glutinosa & 134 \\
\hline & B. bigelovii & 95,111 \\
\hline & B. vaccinoides & 95 \\
\hline & B. salicifolia & 42,95 \\
\hline \multirow[t]{8}{*}{$5,7,4^{\prime}-\mathrm{OH}$ flavanona (naringenina) } & B. alaternoides & 133 \\
\hline & B. illinita & 157 \\
\hline & B. salzmannii & 46 \\
\hline & B. heterophylla & 95 \\
\hline & B. ramosissimum & 47 \\
\hline & B. varians & 46 \\
\hline & B. ligustrina & 109 \\
\hline & B. pseudotenuifolia & 87 \\
\hline 5,7-OH-4'-OMe flavanona & B. alaternoides & 133 \\
\hline \multirow[t]{2}{*}{ (isosakuranetina) } & B. salicifolia & 42,43 \\
\hline & B. vaccinoides & 95 \\
\hline 5,7-OH-4'-OMe flavanona & B. leptocephala & 47 \\
\hline (isosakuranetina) & B. polifolia & 42 \\
\hline 5,7-OH-3'-OMe flavanona & B. truncata & 47 \\
\hline 5,7-OH-3',4'-OMe flavanona & B. confertifolia & 97 \\
\hline 3,5,7-OH flavanona & B. oxydonta & 47 \\
\hline (pinobanksina) & B. bigelovii & 95,111 \\
\hline $3,5,7,4^{\prime}-\mathrm{OH}$ flavanona & B. pseudotenuifolia & 87 \\
\hline (aromadendrina) & B. illinita & 157 \\
\hline $3,5,7,3^{\prime}, 4^{\prime}-\mathrm{OH}$ flavanona & B. petiolata & 97 \\
\hline (taxifolina) & B. illinita & 157 \\
\hline 3,5,7-OH-4'-OMe flavanona & B. dracunculifolia & 159 \\
\hline $\begin{array}{l}\text { 3,5,7-OH-6-OMe flavanona } \\
\text { (alnustinol) }\end{array}$ & B. bigelovii & 43,111 \\
\hline 4'-OH-5,7-OMe flavanona & B. alaternoides & 133 \\
\hline $2 \beta-5,7-\mathrm{OH}$ flavanona & B. bigelovii & 111 \\
\hline 5,3',4'-OH-7-OMe flavanona & B. marginalis & 113 \\
\hline (7-metileriodictiol) & B. paniculata & 97 \\
\hline 5,4'-OH-7-O-prenil flavanona & B. potosina & 43 \\
\hline 3,5,3',4'-OH-7-O-prenil flavanona & B. potosina & 43 \\
\hline $5,7,4^{\prime}-\mathrm{OH}-3-\mathrm{OAc}$ flavanona & B. varians & 46 \\
\hline $\begin{array}{l}5,7,4^{\prime}-\mathrm{OH}-3^{\prime}-\mathrm{OMe}-3-\mathrm{OAc} \\
\text { flavanona }\end{array}$ & B. salicifolia & 42 \\
\hline 5,7,3',4'-OH-3-OAc flavanona & B. varians & 46 \\
\hline Quercetagin-3,6,3'-Me & B. gilliesii & 101 \\
\hline
\end{tabular}

vidade antioxidante em várias espécies de Baccharis. Entre elas destacam-se $B$. trinervis e $B$. coridifolia em inibir a peroxidação lipídica e o sequestro dos radicais hidroxila e superóxido ${ }^{65,66}$, onde tais atividades estão associadas a presença de flavonóides.

\section{CONCLUSÕES}

O gênero Baccharis por sua ampla distribuição geográfica e grande variedade de espécies, associado ao notável destaque na medicina popular no Brasil e em outros países da América do Sul, apresenta estudos de atividade biológica um tanto tímidos. Seu grande potencial em metabólitos secundários e atividades descritas na medicina popular, deixam um vastíssimo campo aberto para a pesquisa de novas moléculas ativas.
A ocorrência de flavonóides aglicônicos é uma característica marcante neste gênero, onde observamos a predominância de flavonas, das quais cerca de $50 \%$ são C-3 oxigenadas. Isto o diferencia na tribo Astereae, onde a ocorrência é de $73 \%$ de flavonas C-3 oxigenadas. A maior percentagem de oxidação na posição 6 , representando um terço da ocorrência de flavonóides em Baccharis, sobre a posição 8, aliada ao alto grau de metoxilação (72\%) vem corroborar com dados da literatura, que coloca a família Asteraceae na condição de um grupo avançado na escala quimioevolutiva.

\section{REFERÊNCIAS}

1. Heywood, V. H.; Flowering plants of the world, Oxford University Press: New York, 1993.

2. Joly, A. B.; Botânica: introdução a taxonomia vegetal, $7^{\mathrm{a}}$ ed., Cia Editora Nacional: São Paulo, 1967.

3. Zomlefer, W. B.; Guide to flowering plant families, Chapel Hill \& London: Carolina, USA, 1994

4. Emerenciano, V. P.; Militão, J. S. L. T.; Campos, C. C.; Romofe, P.; Kaplan, M. A. C.; Zambon, M.; Brant, A. J. C.; Biochem. Syst. Ecol. 2001, 29, 947.

5. Harborne, J. B.; Willians, C. A.; Phytochemistry 2000, 55, 481.

6. Malagarriga Heras, R. D. P.; Mem. Soc. Cienc. Nat. 1976, 23, 129.

7. Dupont, P.; Bull. Soc. Bretogne 1966, 41, 141.

8. Teodoro-Luis, I.; Contr. Inst. Geobiol. (Brasil) 1955, 5, 1.

9. Barroso, G. M.; Rodriguésia 1975-6, $28,3$.

10. Espinar, L.; Bol. Acad. Nac. Cienc. Argentina 1973, 50, 175.

11. Matuda, E.; Ann. Inst. Biol. 1957, 28, 143.

12. Cuatrecasas, J.; Rev. Acad. Colombia Cienc. 1967, 13, 5.

13. Cuatrecasas, J.; Rev. Acad. Colombia Cienc. 1968, 13, 201

14. Gary, A.; Smithsonian Contr. to Knowledge 1853, 5, 80.

15. Gray, A.; Synoptical flora of Norh America, Taylor: New York, 1884.

16. Korbes, C. V.; Manual de plantas medicinais, $48^{\mathrm{a}}$ ed., Grafit: Francisco Beltrão, 1995

17. Franco, I. J.; Ervas e plantas: a medicina dos simples, $5^{\mathrm{a}}$ ed., Imprimax: Chapecó, 1995.

18. Corrêa, M. P.; Dicionário das plantas úteis do Brasil e das exóticas cultivadas, Imprensa Nacional: Rio de Janeiro, 1984, vol. 1-6.

19. Bandoni, A. L.; Medina, J. E.; Rondina, R. V. D.; Coussio, J. D.; Planta Med. 1978, 34, 328.

20. Cortadi, A.; Di Sapio, O.; Mc Cargo, J.; Scandizzi, A.; Gattuso, S.; Gattuso, M.; Pharm. Biol. 1999, 37, 357.

21. Melo, S. F.; Soares, S. F.; Costa, R. F.; Mutat. Res. 2001, 496, 33.

22. Emerenciano, V. P.; Ferreira, Z. S.; Kaplan, M. A. C.; Gottlieb, O. R.; Phytochemistry 1987, 26, 3103.

23. Jakupovic, J.; Schuster, A.; Ganzer, U.; Bohlmann, F.; Boldt, P. E.; Phytochemistry 1990, 29, 2217.

24. Boldt, P. E.; Baccharis (Asteraceae) a review of its taxonomy, phytochemistry, ecology, economic status. College Station, Texas, The Texas A \& M University System, 1989.

25. Tokarnia, C. H.; Peixoto, P. V.; Gava, A.; Barros, C. S. L.; Pesq.Veter. Brasil. 1992, 12,19

26. Jarvis, B. B.; Mokhtari-Rejali, N.; Schenkel, E. P.; Barros, C. S.; Matzenbacher, N. I.; Phytochemistry 1991, 30, 789.

27. Ueno, Y.; Trichotecenes: chemical, biological and toxicological aspects, Kodansa Press: Tokio, 1983

28. Tokarnia, C.; Dobereiner, J.; Pesq. Agropec. Brasil. 1975, 10, 79

29. Rizzo, I.; Varsavky, E.; Haidukowski, M.; Frade, H.; Toxicon 1997, 35, 753.

30. Povolny, D.; Acta. Entomol. Bohemoslovaca 1980, 77, 55.

31. Bailey, E.; Bailey, L.; Hortus Third, MacMillan Company: New York, 1976.

32. Motl, O.; Trka, A.; Porfum. Kostmet. 1983, 64, 488.

33. Jarvis, B. B.; Midiwo, J. O.; J. Nat. Prod. 1988, 51, 736.

34. Kupchan, S. M.; Jarvis, B. B.; Dailey, R. G.; Bright, W.; Bryan, R. F.; Shizuri, Y.; J. Am. Chem. Soc. 1976, 98, 7092.

35. Kupchan, S. M.; Streelman, D. R.; Jarvis, B. B.; Dailey, R. G.; Sneden, A. T.; J. Org. Chem. 1977, 42, 4221.

36. Garcia, C. C.; Rosso, M. L.; Bertoni, M. D.; Maier, M. S., Damonte, E. B.; Planta Med. 2002, 68, 209.

37. Habermehl, G.; Busam, L.; Heydel, P.; Mebs, D.; Tokarnia, C.; Dobereiner, J.; Spraul, M.; Toxicon 1985, 23, 731.

38. Dobereiner, J.; Resende, A.; Tokarnia, C.; Pesq. Agropec. Brasil. 1976, 11, 27.

39. Jarvis, B. B.; Comezoglu, S. N.; Amomon, H. L.; Breedlove, C. K.; Miller, R. W.; Woode, M. K.; Streelman, D. R.; Sneden, A. T.; Dailey, R. G.; Kupchan, S. M.; J. Nat. Prod. 1987, 50, 815. 
40. Jarvis, B. B.; Comezoglu, S. N.; Rao, M. M.; Pena, N. B.; J. Org. Chem 1987, 52,45

41. Jarvis, B. B.; Wells, K. M.; Lees, Y. W.; Bean, G. A.; Kommedahl, T.; Barros, C. S. Phytopathology 1987, 77, 980.

42. Zdero, C.; Bohlmann, F.; King, R. M.; Robinson, H.; Phytochemistry 1986, 25,2841

43. Jakupovic, J.; Schuster, A.; Ganzer, U.; Bohlmann, F.; Boldt, P. E.; Phytochemistry 1990, 29, 2217.

44. Bohlmann, F.; Banerjee, S.; Jalupovic, J.; Grenz, M.; Misra, L. N.; Hirschmann, G. S.; King, R. M.; Robinson, H.; Phytochemistry 1985, 24 511.

45. Hikawczuk, V. E. J.; Rossomando, P. C.; Giordano, O. S., Saad, J. R.; Phytochemistry 2002, 61, 389.

46. Bohlmann, F.; Zdero, C.; Grenz, M.; Dhar, A. K.; Robinson, H.; King, R. M.; Phytochemistry 1981, 20, 281.

47. Bohlmann, F.; Kramp, W.; Grenz, M.; Robinson, H.; King, R. M.; Phytochemistry 1981, 20, 1907.

48. Zdero, C.; Bohlmann, F.; Solomon, J. C.; King, R. M.; Robinson, H.; Phytochemistry 1989, 28, 531.

49. Fullas, F.; Hussain, R. A.; Chai, H-B.; Pezzuto, J. M.; J. Nat. Prod. 1994 $57,801$.

50. Labbe, C.; Castillo, M.; Hernandez, M.; Phytochemistry 1991, 30, 1607.

51. Enriz, E. D.; Baldoni, H. A.; Jauregui, E. A.; Sosa, M. E.; Tonn, C. E.; Luco, J. M.; Gordaliza, M.; J. Agric. Food Chem. 2000, 48, 1384.

52. Torres, L. M. B.; Gamberini, M. T.; Roque, N. F.; Landman, M. T. L.; Souccar, C.; Lapa, A. J.; Phytochemistry 2000, 55, 617.

53. Rahalison, L.; Benathan, M.; Monod, M.; Frenk, E.; Gupta, M. P.; Solis, P. N.; Fuzzati, N.; Hostettmann, K.; Planta Med. 1995, 61, 360.

54. Kupchan, S. M.; Bauerschmidt, E.; Phytochemistry 1971, 10, 664.

55. René, R. M.; Cartaña, C.; Adzet, T.; Marín, E.; Parella, T.; Cañigueral, S.; Planta Med. 1996, 62, 232

56. Nakasugi, T.; Komai, K.; J. Agric. Food Chem. 1998, 46, 2560.

57. Soicke, H.; Leng-Peschlow, E.; Planta Med. 1987, 53, 37.

58. Palacios, P.; Gutkind, G.; Rondina, R. V. D.; Torres, R.; Coussio, J. D.; Planta Med. 1983, 49, 128

59. Cobos, M. I.; Rodriguez, J. L.; Oliva, M. L.; Demo, M.; Faillaci, S. M.; Zygadlo, J. A.; Planta Med. 2001, 67, 84

60. Feresin, G. E.; Tapia, A.; López, S. N.; Zacchino, S. A.; J. Ethnopharm 2001, 78, 103

61. Weimann, C.; Goransson, U.; Pongprayoon-C, U.; Claeson, P.; Bohlin, L.; Rimpler, H.; Heinrich, M.; J. Pharm. Pharmacol. 2002, 54, 99.

62. Cazon, A.; Viana, M.; Gianello, J. C.; Rev. Biol. Trop. 2000, 48, 47.

63. Cespedes, C. L.; Uchoa, A.; Salazar, J. R.; Perich, F.; Pardo, F.; J. Agric Food Chem. 2002, 50, 2283.

64. Sanchez Palomino, S.; Abad, M. J.; Bedoya, L. M.; Biol. Pharm. Bull. 2002, 25, 1147

65. Las Heras de, B.; Slowing, K.; Benedí, J.; J. Ethnopharm. 1998, 61, 161.

66. Mongelli, E.; Desmarchelier, C.; Rodriguez Talou, J.; Coussio, J.; Ciccia, G.; J. Ethnopharm. 1997, 58, 157.

67. Jarvis, B. B.; Midiwo, J. O.; Tuthill, D.; Bean, G. A.; Science 1981, 214 460.

68. Pérez-García, F.; Marín, E.; Adzet, T.; Cañigueral, S.; Phytomedicine 2001 8,31 .

69. Jarvis, B. B.; Pena, N. B.; Rao, M. M.; Comezoglu, N. S.; Comezoglu, T F.; Mandava, N. B.; ACS Symp. Ser. 1985, 268, 149.

70. Feresin, G. E.; Tapia, A. A.; Bustos, D. A.; Fitoterapia 2000, 71, 429.

71. Kuti, J. O.; Jarvis, B. B.; Mokhtarirejali, N.; Bean, G. A.; J. Chem. Ecol. 1990, 16, 3441 .

72. Abad, M. J.; Bermejo, P.; Gonzales, E.; Iglesias, I.; Irurzum, A.; Carrasco, L.; Gen. Pharmacol. 1999, 32, 499.

73. Mongelli, E.; Pampuro, S.; Coussio, J.; Salomon, H.; Ciccia, G.; J. Ethnopharm. 2000, 71, 145.

74. Tortorielli, J.; Aguilar-S, L.; J. Ethnopharm. 1996, 53, 157.

75. Gonzales, E.; Iglesias, I.; Carretero, E.; Villar, A.; J. Ethnopharm. 2000, $70,329$.

76. Argandona, V. H.; Faini, F. A.; Phytochemistry 1993, 33, 1377.

77. Sosa, M. E.; Tonn, C. E.; Giordano, O. S.; J. Nat. Prod. 1994, 57, 1262.

78. Ciccia, G.; Coussio, J.; Mongelli, E.; J. Ethnopharm. 2000, 72, 185.

79. Bergmann, F.; Yagen, B.; J. Toxicol. Toxin Rev. 1993, 12, 91.

80. Bergmann, F.; Yagen, B.; Jarvis, B. B.; Toxicon 1992, 30, 1291.

81. Perez, F.; Marin, E.; Adzet, T.; Phytother. Res. 1995, 9, 145.

82. Cifuente, D. A.; Borkowwski, E. J.; Sosa, M. E.; Gianello, J. C.; Giordano, O. S.; Tonn, C. E.; Phytochemistry 2002, 61, 899

83. Rojas, A.; Bah, M.; Rojas, J. I.; Serrano, V.; Pacheco, S.; Phytomedicine 1999, 6, 367

84. Faini, F.; Labbe, C.; Coll, J.; Biochem. Syst. Ecol. 1999, 27, 673.

85. Marston, A.; Hostettmann, K.; Phytochemistry 1985, 24, 639.
86. Garcia, C. C.; Rosso, M. L.; Bertoni, M. D.; Maier, M. S.; Damonte, E. B.; Planta Med. 2002, 68, 209.

87. Moreira, F. P. M.; Coutinho, V.; Montanher, A. B. P.; Caro, M. S. B.; Brighente, I. M. C.; Pizzolatti, M. G.; Quim. Nova 2003, 26, 309.

88. Abad, M. J.; Bermejo, P.; Palomino, S. S.; Chiriboga, X.; Carrasco, L.; Phytother. Res. 1999, 13, 142.

89. Baggio, C. H.; Freitas, C. S.; Rieck, L.; Marques, M. C. A.; Pharmacol. Res. 2003, 47, 93.

90. Monks, N. R.; Ferraz, A.; Bordignon, S.; Machado, K. R.; Lima, M. F. S.; Rocha, A. B.; Schwartsmann, G.; Pharm. Biol. 2002, 40, 494

91. Desmarchelier, C.; Bermudez, M. J. N.; Coussio, J.; Ciccia, G.; Boveris, A.; Int. J. Pharm. 1997, 35, 116

92. Oliveira, S. Q.; Dal-Pizzol, F.; Gosmann, G.; Guillaume, D.; Moreira, J. C. F.; Schenkel, E. P.; Free Radical Res. 2003, 37, 555.

93. Charp, H.; Bartholomew, B.; Bright, C.; Latif, Z.; Sarker, S. D.; Nash, R. J.; Biochem. Syst. Ecol. 2001, 29, 105.

94. Mendez, A. M.; Nunez, O.; Rosquete, C.; Montilla, A.; Ann.Quim. S. CQuim. Org. y Bioq.1984, 80, 98.

95. Wollenweber, E.; Schober, I.; Dostal, P.; Hradetzky, D.; Z. Naturforsch.C: J. Biosci. 1986, 41, 87.

96. Akaike, S.; Sumino, M.; Sekine, T.; Seo, S.; Kimura, N.; Ikegami, F.; Chem. Pharm. Bull. 2003, 51, 197.

97. Faini, C. L. F.; Castillo, M.; Phytochemistry 1990, 29, 324 e ref. citadas.

98. Parodi, F. J.; Fischer, N. H.; Phytochemistry 1988, 27, 2987.

99. Quijano, L.; Rios, T.; Fronczek, F. R.; Phytochemistry 1998, 49, 2065.

100. Rivera, A. P.; Faini, F.; Castillo, M.; J. Nat. Prod. 1988, 51, 155.

101. Gianello, J. C.; Giordano, O. S.; Rev. Latinamer. Quim. 1984, 15, 84

102. Arriaga Giner, F. J.; Del Castillo, J. B.; Ferrero, M. T. M.; Pena de Recinos, S.; Rodriguez, F. L.; Rev. Latinamer. Quim. 1982, 13, 47.

103. Saad, J. R.; Davicino, J. G.; Giordano, O. S.; Phytochemistry 1987, 27, 1884.

104. Gianello, J. C.; Tonn, C. D.; Kavka, J.; Guerreiro, E.; Giordano, O. S.; Abstract, XIV Congresso Latinoamericano de Ouimica, Costa Rica, 1981.

105. Kuroyanagi, M.; Fujita, K.; Kazaoka, M.; Matsumoto, S.; Ueno, A.; Fukushima, S.; Katsuoka, M.; Chem. Pharm. Bull. 1985, 33, 5075.

106. He, K.; Montenegro, G.; Hoffmann, J. J.; Timmermann, B. N.; Phytochemistry 1996, 41, 1123.

107. Cordano, G. F.; Peña de La, A. M.; Medina, J. J.; J. Nat. Prod. 1982, 45, 653.

108. Daily, A.; Wagner, H.; Seligmann, O.; Fitoterapia 1984, 55, 236.

109. Moreira, F. P. M.; Branco, A.; Pizzolatti, M. G.; Morais, A. A.; Monache, F. D.; Biochem. Syst. Ecol. 2003, 31, 319

110. Liu, Y-L.; Timmermann, B. N.; Hoffmann, J. J.; McLaughlin, S. P.; Phytochemistry 1993, 33, 1549.

111. Arriaga-Giner, F. J.; Z. Naturforsch. C: J. Biosci. 1986, 41, 946.

112. Moreira, E. A.; Keller, C. C.; Krarmbeck, R.; Miguel, O. G.; Phytochem. Anal. 1975, 43, 3.

113. Faini, F.; Rivera, P.; Mahu, M.; castillo, M.; Phytochemistry 1987, 26, 3281.

114. San Martin, A.; Rovirosa, J.; Castillo, M.; Bol. Soc. Chil. Quim. 1982, 127, 252.

115. Suttisri, R.; Kinghorn, A. D.; Wright, A. D.; Sticher, O.; Phytochemistry 1994, 35, 443.

116. Mesquita, A. A. L.; Correa, D. B.; Pádua, A. P.; Guedes, M. L. O.; Gottlieb, O. R.; Phytochemistry 1986, 25, 1255

117. Tonn, C. E.; Rossomando, P. C.; Giordano, O. S.; Phytochemistry 1982, $21,2599$.

118. Bohlmann, F.; Kramp, W.; Jakupovic, J.; Robinson, H.; King, R. M.; Phytochemistry 1982, 21, 399.

119. Faini, F.; Labbe, C.; Polanco, M. I.; Phytochemistry 1992, 31, 3274.

120. Zdero, C.; Bohlmann, F.; Niemeyer, H. M.; Phytochemistry 1991, 30, 1597.

121. Bohlmann, F.; Zedero, C.; King, R. M.; Robinson, H.; Phytochemistry 1983, 23, 1511 .

122. Herz, W.; Pilotti, A. M.; Söderholm, A. C.; Shuhama, I. K.; Vichnewski, W.; J. Org. Chem. 1977, 42, 3913.

123. Herrera, J. C.; Romero, A. J. R.; Crescente, O. E.; Acosta, M.; Pekerar, S.; J. Chromatogr. 1996, 740, 201.

124. Jakupovic, J.; Schuster, A.; Wasshausen, D. C.; Phytochemistry 1991, 30, 2785.

125. San Martin, A.; Rovirova, J.; Castillo, M.; Phytochemistry 1983, 22, 1461.

126. Silva, M.; Mundaca, J. M.; Phytochemistry 1971, 10, 1942.

127. Saad, J. R.; Pestchanker, M. J.; Giordano, O. S.; Phytochemistry 1987, 26, 3033.

128. Faini, F. A.; Castillo, M.; Torres, M. R.; J. Nat. Prod. 1982, 45, 501

129. Silva, G. A. B.; Henriques, A.; Alice, C. B.; Gleye, J.; Moulis, C.; J. Nat. Prod. 1985, 48, 861.

130. Schenkel, E. P.; Sobottka, A. M.; Lankhorst, P. P.; Schripsema, J.; Phytochemistry 1997, 44, 755. 
131. Wagner, H.; Iyengar, M. A.; Phytochemistry 1972, 11, 444.

132. Bohlmann, F.; Scheidges, C.; Zdero, C.; King, R. M.; Robinson, H.; Phytochemistry 1984, 23, 1109.

133. Bohlmann, F.; Knauf, W.; King, R. M.; Robinson. H.; Phytochemistry 1979, $18,1011$.

134. Miyakado, M.; Kato, T.; Ohno, N.; Mabry, T. J.; Phytochemistry 1979, 15, 846.

135. Tonn, C. E.; Giordano, O. S.; Martín, J. D.; Martín, V. S.; Phytochemistry 1989, 28, 1537.

136. Tonn, C. E.; Giordano, O. S.; Bessalle, R.; Frolow, F.; Lavie, D.; Phytochemistry 1988, 27, 489.

137. Dai, J.; Suttisri, R.; Bordas, E.; Soejarto, D. D.; Kinghorn, A. D.; Phytochemistry 1993, 34, 1087.

138. Stapel, G.; Menssen, H. G.; Snatzke, G.; Planta Med. 1980, 39, 366.

139. Cenal, J. P.; Giordano, O. S.; Rossomando, P. C.; Tonn, C. E.; J. Nat. Prod. 1997, 60, 490 .

140. Fullas, F.; Soejarto, D. D.; Kinghorb, A. D.; Phytochemistry 1992, 31, 2543.

141. Fullas, F.; Hussain, R. A.; Bordas, E.; Pezzuto, J. M.; Tetrahedron 1991, 47, 8515 .

142. Arriaga-Giner, F. J.; Wollenweber, E.; Schober, I.; Dostal, P.; Braun, S.; Phytochemistry 1986, 25, 719.

143. Givovich, A.; San-Martín, A.; Castillo, M.; Phytochemistry 1986, 25, 2829.

144. San-Martín, A.; Givovich, A.; Castillo, M.; Phytochemistry 1986, 25, 264.

145. Brown, G. D.; Phytochemistry 1994, 35, 1037.

146. Gambaro, V.; Chamy, M. C.; Garbarino, J. A.; Phytochemistry 1987, 26, 475 .
147. Gambaro, V.; Chamy, M. C.; Garbarino, J. A.; San-Martín, A.; Castillo, M.; Phytochemistry 1986, 25, 2175.

148. Cifuente, D. A.; Tonn, C. E.; Giordano, O. S.; Nat. Prod. Lett. 2001, 15, 425.

149. Li, C-J.; Ahmed, A. A.; Arias, A. D. C.; Mabry, T. J.; Phytochemistry 1997, $45,571$.

150. Wachter, G. A.; Montenegro, G.; Timmermann, B. N.; J. Nat. Prod. 1999, $62,307$.

151. Zdero, C.; Bohlmann, F.; Niemeyer, H. M.; Phytochemistry 1990, 29, 2611.

152. Moreira, F. P. M.; Dissertação de Mestrado, Universidade Federal de Santa Catarina, Brasil, 2000

153. San-Martín, A.; Rovirosa, J.; Labbé, C.; Givovich, A.; Mahú, M.; castillo, M.; Phytochemistry 1986, 25, 1393.

154. San Martín, A.; Rovirosa, J.; Becker, R.; Castillo, M.; Phytochemistry 1980, 19, 1985.

155. Kuroyanagi, M.; Uchida, K.; Ueno, A.; Satake, M.; Shimomura, K.; Phytochemistry 1993, 34, 1377.

156. Rossomando, P. C.; Giordano, O. S.; Espiñeira, J.; Joseph-Nathan, P.; Phytochemistry 1985, 24, 787.

157. Verdi, L. G.; Brighente, I. M. C.; Schripsema, J.; Braz Filho, R.; Pizzolatti, M. G.; Biochem. Syst. Ecol. 2004, 32, ??.

158. Feresin, G. E.; Tapia, A.; Gimenez, A.; Ravelo, A. G.; Zacchino, S.; Sortino, M.; Hirschmann, G. S.; J. Ethnopharm. 2003, 89, 73.

159. Kumazawa, S.; Yoneda, M.; Shibata, I.; Kanaeda, J.; Hamasaka, T.; Nakayama, T.; Chem. Pharm. Bull. 2003, 51, 740. 